\title{
Tunnelling Effect for Quadruples Potential Using Matrix Propagation Method
}

\author{
Chilwatun Nasiroh*, Bambang Supriadi, and Rif'ati Dina Handayani \\ Physics Education Department, University of Jember \\ Jl. Kalimantan No.37, Krajan Timur, Sumbersari, Jember, Indonesia \\ *Email: chilwatunnasiroh25@gmail.com
}

\begin{tabular}{|c|c|}
\hline Article Info & ABSTRACT \\
\hline $\begin{array}{l}\text { Article History } \\
\text { Received Nov 5, } 2020 \\
\text { Accepted Nov 28, } 2020 \\
\text { Published Dec 31, } 2020\end{array}$ & $\begin{array}{l}\text { Semiconductor materials can be used as potential barriers to Tunnelling effects. In } \\
\text { this study, four semiconductor materials are arranged in various ways to form a } \\
\text { quadruple potential structure to analyze the value of the transmission coefficient. The } \\
\text { analysis was conducted using the analytical and numerical matrix propagation method }\end{array}$ \\
\hline $\begin{array}{l}\text { Keywords: } \\
\text { Transmission coefficient } \\
\text { Tunneling effect } \\
\text { Semiconductor }\end{array}$ & $\begin{array}{l}\text { same transmission coefficient value for each energy. So that there are } 12 \text { kinds of } \\
\text { transmission coefficient values generated from } 24 \text { arrangements. The semiconductor } \\
\text { material composition with the most considerable transmission coefficient value is } \\
\text { ADCB and BCDA, which have a value of } 0.8087 \text {. The variation of the arrangement } \\
\text { affects the value of the transmission coefficient so that it can be used as a guideline } \\
\text { for selecting the arrangement that produces the most optimum value of the } \\
\text { transmission coefficient from various possible arrangements. }\end{array}$ \\
\hline
\end{tabular}

To cite this article:

C. Nasiroh, B. Supriadi, and R. D. Handayani, "Tunnelling Effect for Quadruples Potential Using Matrix Propagation Method," Indones. Rev. Phys., vol. 3, no. 2, pp. 66-73, 2020.

\section{Introduction}

Previously, scientists believed that all the natural phenomena could be solved using Newtonian mechanics and Maxwell electromagnetics. Until the end of the 19th century, the belief that classical physics could explain all natural phenomena began to diminish when scientists discovered several new phenomena that could not be explained by classical physics concepts. This phenomenon mainly appears in microscopic physical objects such as elementary and atomic particles [1].

One of the interesting phenomena in quantum mechanics is the Tunnelling effect. The Tunnelling effect occurs when the particles pass through a barrier with an energy higher than the particles' energy. According to classical mechanics, particles with energy that propagate to the right and encounter a potential, then particles will be reflected the left [1]. Nevertheless, quantum mechanics believe that particles can pass through barriers. The ability of particles to penetrate this barrier is due to the wave's nature, which plays a more critical role than the nature of particles [2]. Particles have the probability of being able to break through a barrier even though the energy is less than the energy of the barrier. The magnitude of this probability is called the transmission coefficient [2].

The transmission coefficient can be analyzed using several methods, namely the variable separation method in the Schrodinger equation, the Propagation Matrix, and the WKB (Wentzel-Kramers-Brillouin). In this study, the transmission coefficient will be analyzed using the matrix propagation method because it is easy to use in cases with a relatively large number of obstructions. Abdulhalim's research on some layered structures' optical properties shows that the analytical expression derived from the matrix propagation method is more comfortable to apply than using direct computation [3]. The use of the matrix propagation method is divided into three main steps: first, calculating the propagation matrix to transmit a particle's wave function with energy $\mathbb{E}$ moving towards a single potential barrier. Second, compute the propagation matrix for the wave function between potential steps. Third, calculate the barrier's total propagation by multiplying each propagation of each potential [4].

The transmittance of electrons through potential barriers is a research topic that has caught by many researchers'. Martinz and Ramos [5] determine the transmission coefficient's value analytically and numerically on the potential triple barrier with two potential wells. Research on the potential triple barrier of $\mathrm{GaN}, \mathrm{SiC}$, and GaAs materials by Supriadi has a maximum transmission coefficient value of 0.819 [6], and Prastowo's research [7] on the potential triple barrier of Graphene material obtained a resonance phenomenon with the most considerable transmission coefficient value is 1.0000 at an energy of $0.9200 \mathrm{eV}$.

Research on the effects of breakthroughs has contributed to developing electronic devices on the nanometer scale with increasingly impressive performance. Electronic devices use semiconductor materials as their main components. Each semiconductor material has characteristics of the size of the potential barrier or energy gap. The research aims to determine the transmission coefficient's value of Semiconductor materials of GaAs, GaSb, AlAs, and InP that arranged 
with several variations to form a quadruple potential barrier structure.

\section{Theory \\ Waves of Particles}

Maxwell's theory of light as a wave and electromagnetic theory flourished well into the late 19th century. However, Einstein in 1905 rejected the theory based on the phenomenon of the photoelectric effect. According to Einstein, light must be seen as a quantum particle called the photon. The energy of the photon is $E=h f$, and momentum is

$p=\frac{h f}{c}=\frac{h}{\lambda}$

This momentum characterizes the particle properties of light [8].

In 1924, Louis de Broglie proposed the hypothesis that particles have waves. A particle with momentum $p$ has a wavelength called the de Broglie wavelength,

$\lambda=\frac{h}{p}$

The wave package fills a quantity capable of accommodating and representing the particles' and the wave's properties. The wave package is approached by combining two plane waves so that the wave function can be represented as follows:

$\Psi(x, t)=N \int \varphi(p) e^{i(p x-E n) h} d p$

$\mathrm{N}$ is the normalization constant [9].

\section{Schrodinger's Equation}

Erwin Schrodinger introduced the equation in 1926 to discuss particle waves' description in the atomic dimension that meets physics's principles and laws [1]. The form of the Schrodinger equation is a second-order partial differential equation. Solving the Schrodinger equation is by applying the variable separation method to get the solution.

The Schrodinger equation must obey the law of energy conservation since the total energy- the kinetic energy and the potential energy of a particle is conserved. Besides, the Schrodinger equation must comply with de Broglie's postulate; the mathematical solution for a particle with momentum $p$ must be in the form of a wave function with $\lambda$ the same wavelength as $h / p$.

Solving the Schrodinger equation must provide information about the probability of finding the particle that must exist in space. The wave function must be single and finite; otherwise, a particle's existence would be more than one possibility. The wave function must be continuous; it can be interpreted as a breakdown of the particles not to be accepted. The wave function's derivative concerning the position must also be continuous because it is related to the electron's momentum. The indicator of the nature of a well-behaved wave must have a wave superposition characteristic [10].

$\frac{\partial^{-} \Psi}{\partial x^{2}}+\frac{2 m}{\hbar^{2}}(E-V) \Psi=0$

\section{Tunnelling Effect}

The Tunnelling effect is a phenomenon in quantum physics when energetic particles $E$ pass through a potential region $V$ of a barrier on condition $E<V$ (Figure 1).

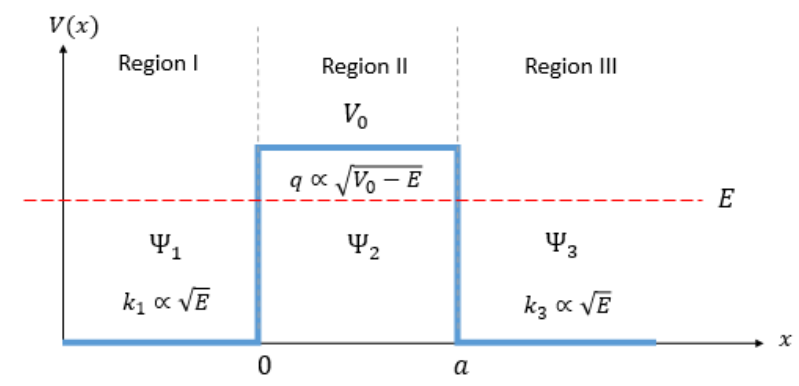

Figure 1. Single potential barrier model $(E \leqslant$ v)

In region $\mathrm{I}(x<0)$, the particles act as free particles because they are not affected by any forces $V(x)=0$. The Schrodinger equation can be written:

$\frac{\partial^{2} \Psi_{1}}{\partial x^{2}}+\frac{2 m E}{h^{2}} \Psi_{1}=0$

or

$\frac{\partial^{2} \Psi_{1}}{\partial x^{2}}=-k_{1}^{2} \Psi_{1}$

with

$k_{1}=\frac{1}{h} \sqrt{2 m E}$

the equation has a solution:

$\Psi_{1}=A e^{i k_{1} x}+B e^{-i k_{1} x}$

Figure 1 represented that the free particle moves from left to right when the particle enters the $0 \leq x \leq a$ area, the particle encounters a potential equal to $V=V_{0}$, so the Schrodinger equation can be written:

$\frac{\partial^{2} \Psi_{2}}{\partial x^{2}}+\frac{2 m\left(E-V_{0}\right)}{h^{2}} \Psi_{2}=0$

or 
$\frac{\partial^{2} \Psi_{2}}{\partial x^{2}}=-k_{2}^{2} \Psi_{2}$

with

$k_{2}=\frac{1}{h} \sqrt{2 m\left(E-V_{0}\right)}$

Since $V_{0}>E_{\mathrm{SO}}, k_{2}=\frac{1}{2 m\left(E-V_{0}\right)}$ it can be written $k_{z}=\frac{1}{h} \sqrt{2 m\left(V_{0}-E\right)}$ or $k_{2}=i q ; q=\frac{1}{h} \sqrt{2 m\left(V_{0}-E\right)}$.

The equation above has a solution:

$\Psi_{2}=C e^{4 x}+D e^{-4 x}$

When the particle manages to break through $x>a$, the particle returns to a free particle $V=0$ so that the Schrodinger equation can be written:

$\frac{\partial^{2} \Psi_{3}}{\partial x^{2}}+\frac{2 m E}{h^{2}} \Psi_{3}=0$

or

$\frac{\partial^{2} \Psi_{3}}{\partial x^{2}}=-k_{3}^{2} \Psi_{3}$

with

$k_{3}=k_{1}=\frac{1}{h} \sqrt{2 m E}$

It appears that the wavenumber of the particle returns to its original state and has a solution:

$\Psi_{n}=F e^{i k_{1} x}$

The transmission coefficient can be defined as the probability that a particle can break through a potential barrier. The equation that can be used to obtain the value of the transmission coefficient on the breakthrough effect is:

$T=|t|^{2}=\left|\frac{F}{A}\right|^{2} \frac{v_{F}}{v_{A}}$

Since the region I and III are the same, that is $V=0$, so $v_{A}=v_{F}$ and $T=\left|\frac{F}{A}\right|^{2}$.

With a mathematical solution using the elimination and substitution method, it is obtained:

$T=\left(1+\left(\frac{k_{1}^{2}+q^{2}}{2 k_{1} q}\right)^{2} \sinh ^{2}(q L)\right)^{-1}$

\section{Matrix Propagation Method}

The matrix propagation method is the spread or transmission of a wave using a matrix. The work with the matrix propagation method is divided into four parts, as ilustrated in the Figure 2 [4].

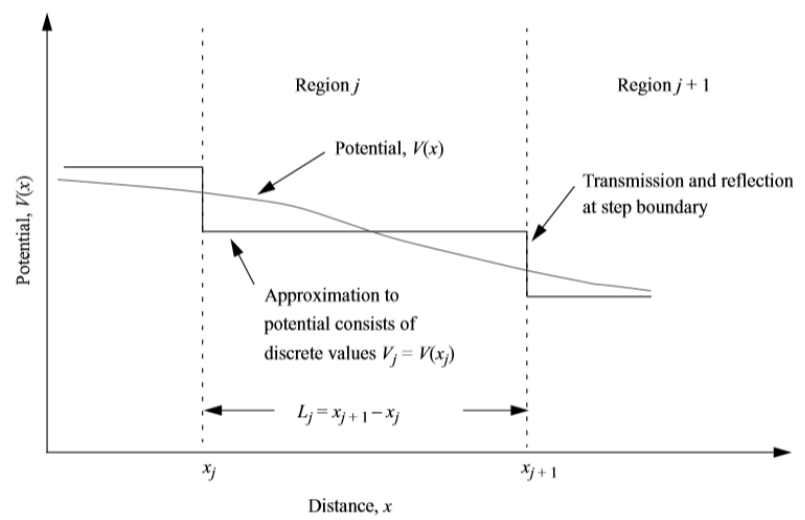

Figure 2. Matrix propagation method

First part: calculate the propagation matrix $\hat{p}_{\text {stap }}$ for transmission and reflection and the wave function representing an energized particle $\mathbb{E}$ entering a ladder potential. The potential ladder in question is in the position $x_{j+1}$ in Figure 2. In the area $j+1$, the particles have a wave number $k_{j}=\frac{\left(2 \mathrm{~m}\left(\bar{E}-\theta \mathrm{V}_{j}\right)^{\mathrm{I} / 2}\right.}{h}$ and a wave function

$\psi_{j}=A_{j} e^{i k_{j} x}+B_{j} e^{-i k_{j} x}$

$\psi_{j+1}=C_{j+1} e^{i h_{j+1} x}+D_{j+1} e^{-i \bar{F}_{j+1} x}$

Applying boundary conditions in the area $j$ and $j+1$, at $x_{j+1}=0$ matrix equation can be written

$\left[\begin{array}{cc}1 & 1 \\ 1 & -1\end{array}\right]\left[\begin{array}{l}A_{j} \\ B_{j}\end{array}\right]=\left[\begin{array}{cc}1 & 1 \\ k_{j+1} & -\frac{k_{j+1}}{k_{j}}\end{array}\right]\left[\begin{array}{l}C_{j+1} \\ D_{j+1}\end{array}\right]$

This equation can be reduced to

$\left[\begin{array}{l}A_{j} \\ B_{j}\end{array}\right]=p_{\text {srap }}\left[\begin{array}{l}C_{j+1} \\ D_{j+1}\end{array}\right]$

$p_{\text {jiap }}$ are a two $\times$ two matrices describing the wave propagation at the ladder potential $j$, with the value ie

$\hat{p}_{\text {inap }}=\frac{1}{2}\left[\begin{array}{cc}1+\frac{k_{j+1}}{k_{j}} & 1-\frac{k_{j+1}}{k_{j}} \\ 1-\frac{k_{j+1}}{k_{j}} & 1+\frac{k_{j+1}}{k_{j}}\end{array}\right]$

Second part: calculate the propagation matrix $\hat{p}_{\text {free }}$ for the propagation of the wave function between two ladder potentials. The propagation in question is between $x_{j}$ and $x_{j+1}$. In Figure 2, the width of this propagation is 
$L_{j}=x_{j+1}-x_{j}$. The propagation between the potential ladder with the potential width $L_{j}$ carries information about the phase so that it is in the form of a matrix i.e.

$\left[\begin{array}{cc}e^{i k_{j} L_{j}} & 0 \\ 0 & e^{-i k_{j} z_{j}}\end{array}\right]\left[\begin{array}{l}A_{j} \\ B_{j}\end{array}\right]=\left[\begin{array}{l}C_{j+1} \\ D_{j+1}\end{array}\right]$

$\left[\begin{array}{l}A_{j} \\ B_{j}\end{array}\right]=p_{j \mathrm{rax}}\left[\begin{array}{c}C_{j+1} \\ D_{j+1}\end{array}\right]$

$\hat{p}_{j / \mathrm{rzz}}=\left[\begin{array}{cc}e^{-i k_{j} L_{j}} & 0 \\ 0 & e^{i k_{j} z_{j}}\end{array}\right]$

The propagation of the matrix over the area $;$ consists of free area propagation and ladder propagation.

$p_{j}=p_{j \mathrm{rag}} p_{j \mathrm{kap}}=\left[\begin{array}{ll}p_{11} & p_{12} \\ p_{21} & p_{22}\end{array}\right]$
$p_{j}=\frac{1}{2}\left[\begin{array}{ll}\left(1+\frac{k_{j+1}}{k_{j}}\right) e^{-k_{j} z_{j}} & \left(1-\frac{k_{j+1}}{k_{j}}\right) e^{-i k_{j} L_{j}} \\ \left(1-\frac{k_{j+1}}{k_{j}}\right) e^{i k_{j} L_{j}} & \left(1+\frac{k_{j+1}}{k_{j}}\right) e^{i k_{j} L_{j}}\end{array}\right]$

$p_{11}=p_{22}^{*}$ and $p_{21}=p_{12}^{*}$

With this symmetry, it can be written

$p_{j}=\left[\begin{array}{ll}p_{11} & p_{12} \\ p_{12}^{*} & p_{11}^{*}\end{array}\right]$

The fourth part: calculate the total propagation matrix $p$ for the total ladder potential by multiplying all the propagations of each potential area i.e.

$\hat{P}=\hat{p}_{1} \hat{p}_{2} \ldots \hat{p}_{j} \ldots \hat{p}_{N}=\prod_{j=1}^{j=N} \hat{p}_{j}$

When particles come from the left, then $A=1$ and if no particles are reflected from the right then $D=0$.

$$
\begin{aligned}
& {\left[\begin{array}{l}
A \\
B
\end{array}\right]=\left(\prod_{j=1}^{j=N} p_{j}\right)\left[\begin{array}{l}
C \\
D
\end{array}\right]=p\left[\begin{array}{l}
C \\
D
\end{array}\right]} \\
& \left.\left[\begin{array}{l}
1 \\
B
\end{array}\right]=\mid \begin{array}{ll}
p_{11} & p_{12} \\
p_{12}^{*} & p_{11}^{*}
\end{array}\right]\left[\begin{array}{l}
C \\
0
\end{array}\right] \\
& |C|^{2}=\left|\frac{1}{p_{11}}\right|^{2}
\end{aligned}
$$

\section{Semiconductors as Potential Barriers}

A semiconductor is a material with electrical conductivity between the insulator and the conductor [11]. There is an empty area between the valence band and the conduction band called the bandgap energy in semiconductor materials. If the electrons in the semiconductor material have a high enough energy, they can jump from the valence band to the conduction band through the energy gap or forbidden band. These electrons leave an empty quantum state in the valence band called holes. The motion of electrons and holes in semiconductor materials is the same as free particles' motion [12]. Every semiconductor material has a different energy gap at a specific temperature [13]. In this research, the semiconductor material used is a type of III$\mathrm{V}$ semiconductor alloy. The semiconductor materials include GaAs, GaSb, AlAs, and InP.

$\mathrm{GaAs}$ is a material with great potential for electronic and optoelectronic device applications because of its high electron mobility. GaAs is typically used for laser diodes and high-speed transistors. GaAs has an energy bandgap structure with a direct transition (direct bandgap) of 1.424 $\mathrm{eV}$ with a width of $0.565 \mathrm{~nm}$ [14].

$\mathrm{GaSb}$ is an III-V semiconductor alloy with a direct bandgap with an energy band gap of $0.721 \mathrm{eV}$ at room temperature [15] with a width of $0.610 \mathrm{~nm} \mathrm{[16].}$ Antimony-based alloy semiconductors have relatively high carrier mobility properties. With these properties, this material is interesting to be applied in electronic and optoelectronic devices such as lasers, infrared detectors, magnetic sensors, and high-speed switching devices [17].

AlAs is an III-V semiconductor alloy that is widely applied to optoelectronic devices such as laser diodes. AlAs has a reasonably large energy bandgap of about $2.95 \mathrm{eV}$ [16] with a width of $0.566 \mathrm{~nm}$ [18]. AlAs has less induced voltage, allowing high-performance electrons high mobility so that AlAs can also be applied to HEMT (High Electron Mobility Transistor) transistors [19].

InP is an III-V semiconductor alloy. InP has high electron mobility, so it is widely used for high-powered and high-frequency electronic devices such as pseudomorphic heterojunction bipolar transistors operating at $604 \mathrm{GHz}$. InP has a lattice constant of 0.587 $\mathrm{nm}$ and a direct energy bandgap of $1.29 \mathrm{eV}$, so it can also be applied to optoelectronic devices such as laser diodes [20].

\section{Method}

The semiconductor materials used as a potential barrier are Gallium Arsenide (GaAs), Gallium Antimonide (GaSb), Aluminum Arsenide (AlAs), and Indium Phosphide (InP), each of which has a potential energy of $0.721 \mathrm{eV}, 1.424 \mathrm{eV}, 2.95 \mathrm{eV}$, and $1.29 \mathrm{eV}$. The quantum particle such as an electron tries to pass through the potential barrier with energies in the range $0 \leq E \leq 1$ $\mathrm{eV}$. The potential barriers are separated from each other by a gap of $1 \mathrm{~nm}$ width.

The mathematical method used to determine the transmission coefficient is the matrix propagation method. The matrix propagation method is the spread or transmission of a wave using a matrix. The analytical calculates the free particles' transmission coefficient when experiencing a breakthrough effect using the matrix propagation method. In comparison, numerical is carried 
out by applying the matrix propagation method to the Matlab2018a software.

In the matrix propagation method, the potential area is divided into two parts: the ladder propagation ( $\left.\hat{p}_{s t a g}\right)$ and the free propagation $\left(\hat{\beta}_{\text {fres }}\right) \cdot \beta_{\text {stap }}$ is the propagation in the area of potential change. Meanwhile, $\beta_{\text {free }}$ it is propagation in areas with a constant potential. The ladder propagation $\hat{p}_{\text {step }}$ is divided into step up propagation and step down propagation. Propagation at the boundary from $V=0$ to $V=V_{j}$ is called step-up propagation $\left(p_{\text {step w }}\right)$. Propagation at the boundary from $V=V_{j}$ towards $V=0$ is called step-down propagation ( $\hat{p}_{\text {step down }}$ ). The free propagation $\left(\hat{p}_{\text {fres }}\right)$ is also divided into two as well as $\hat{p}$ step

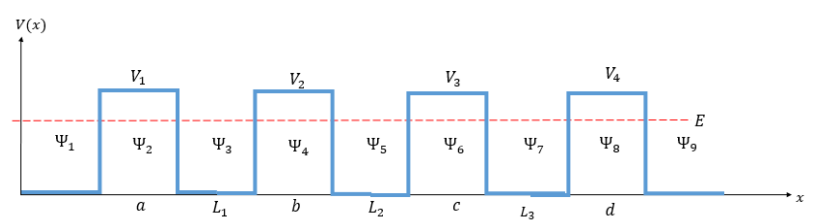

Figure 3. Quadruple potential barrier

The solution to the Schrodinger equation for the potential change in the quadruple barrier from Figure 3 is:

$$
\begin{aligned}
& \Psi_{1}(x)=\frac{A}{\sqrt{k_{1}}} e^{k_{1} x}+\frac{B}{\sqrt{k_{1}}} e^{-i h_{1} x} \\
& \Psi_{2}(x)=\frac{C}{\sqrt{k_{2}}} e^{k_{2} x}+\frac{D}{\sqrt{k_{2}}} e^{-k_{2} x} \\
& \Psi_{3}(x)=\frac{F}{\sqrt{k_{1}}} e^{i k_{1} x}+\frac{G}{\sqrt{k_{1}}} e^{-i k_{1} x} \\
& \Psi_{4}(x)=\frac{H}{\sqrt{k_{3}}} e^{k_{3} x}+\frac{I}{\sqrt{k_{3}}} e^{-k_{3} x} \\
& \Psi_{5}(x)=\frac{J}{\sqrt{k_{1}}} e^{i h_{1} x}+\frac{Z}{\sqrt{k_{1}}} e^{-i k_{1} x} \\
& \Psi_{6}(x)=\frac{M}{\sqrt{k_{4}}} e^{k_{4} x}+\frac{N}{\sqrt{k_{4}}} e^{-k_{4} x} \\
& \Psi_{7}(x)=\frac{0}{\sqrt{k_{1}}} e^{i k_{1} x}+\frac{P}{\sqrt{k_{1}}} e^{-i k_{1} x} \\
& \Psi_{\mathrm{g}}(x)=\frac{5}{\sqrt{k_{5}}} \theta^{k_{\mathrm{B}} x}+\frac{U}{\sqrt{k_{5}}} \theta^{-k_{\mathrm{n}} x} \\
& \Psi_{9}(x)=\frac{V}{\sqrt{k_{1}}} e^{i h_{1} x}+\frac{W}{\sqrt{k_{1}}} e^{-i k_{1} x}
\end{aligned}
$$

The propagation of the quadruple potential barrier consists of four steps up propagations, four free propagations, three propagations in the gap between the step-up potential, and four steps down propagations with the values:

$$
p_{\text {step up } 1}=\frac{1}{2 \sqrt{k_{1} k_{2}}}\left[\begin{array}{ll}
k_{1}-i k_{2} & k_{1}+i k_{2} \\
k_{1}+i k_{2} & k_{1}-i k_{2}
\end{array}\right]
$$

$$
\begin{aligned}
& p_{\text {stap up } z}=\frac{1}{2 \sqrt{k_{1} k_{3}}}\left[\begin{array}{ll}
k_{1}-i k_{3} & k_{1}+i k_{3} \\
k_{1}+i k_{3} & k_{1}-i k_{2}
\end{array}\right] \\
& \hat{p}_{\text {step up a }}=\frac{1}{2 \sqrt{k_{1} k_{4}}}\left[\begin{array}{ll}
k_{1}-i k_{4} & k_{1}+i k_{4} \\
k_{1}+i k_{4} & k_{1}-i k_{4}
\end{array}\right] \\
& \hat{p}_{\text {step up } 4}=\frac{1}{2 \sqrt{k_{1} k_{5}}}\left[\begin{array}{ll}
k_{1}-i k_{5} & k_{1}+i k_{5} \\
k_{1}+i k_{5} & k_{1}-i k_{5}
\end{array}\right] \\
& p_{\text {free } 1}=\left[\begin{array}{cc}
e^{-k_{2 a}} & 0 \\
0 & e^{k_{\text {Za }} a}
\end{array}\right] \\
& \beta_{\text {frea }}=\left[\begin{array}{cc}
e^{-i h_{1} L_{L}} & 0 \\
0 & e^{i k_{1} L_{i}}
\end{array}\right] \\
& \hat{p}_{\text {free } a}=\left[\begin{array}{cc}
e^{-h_{2} b} & 0 \\
0 & e^{h_{Z} b}
\end{array}\right] \\
& p_{\text {froe } 4}=\left[\begin{array}{cc}
e^{-i k_{1} z_{z}} & 0 \\
0 & e^{i k_{1} z_{z}}
\end{array}\right] \\
& \hat{p}_{\text {fres }}=\left[\begin{array}{cc}
e^{-h_{4} e} & 0 \\
0 & e^{i k_{1} c}
\end{array}\right] \\
& \hat{p}_{\text {free }}=\left[\begin{array}{cc}
e^{-i k_{1} z_{3}} & 0 \\
0 & e^{i k_{1} L_{3}}
\end{array}\right] \\
& p_{\text {free }}=\left[\begin{array}{cc}
e^{-k_{5} d} & e^{i} \\
0 & e^{k_{3} d}
\end{array}\right] \\
& \hat{p}_{\text {step dow } 1}=\frac{1}{2 \sqrt{k_{1} k_{2}}}\left[\begin{array}{ll}
k_{2}+i k_{1} & k_{2}-i k_{1} \\
k_{2}-i k_{1} & k_{2}+i k_{1}
\end{array}\right] \\
& p_{\text {stap dow } 2}=\frac{1}{2 \sqrt{k_{1} k_{2}}}\left[\begin{array}{ll}
k_{3}+i k_{1} & k_{3}-i k_{1} \\
k_{3}-i k_{1} & k_{3}+i k_{1}
\end{array}\right] \\
& p_{\text {step dow a }}=\frac{1}{2 \sqrt{k_{1} k_{4}}}\left[\begin{array}{ll}
k_{4}+i k_{1} & k_{4}-i k_{1} \\
k_{4}-i k_{1} & k_{4}+i k_{1}
\end{array}\right] \\
& \hat{p}_{\text {step down } 4}=\frac{1}{2 \sqrt{k_{1} k_{5}}}\left[\begin{array}{ll}
k_{5}+i k_{1} & k_{5}-i k_{1} \\
k_{5}-i k_{1} & k_{5}+i k_{1}
\end{array}\right]
\end{aligned}
$$

So that the propagation of each barrier and each gap can be written:

$\hat{P_{1}}=p_{\text {tap up } 1} \cdot \hat{P}_{\text {free } 1} \cdot \hat{p}_{\text {atop dom } 1}$

$\hat{P}_{\text {gap } 1}=\hat{p}_{\text {free }}$

$\hat{P}_{2}=p_{\text {stop wp } 2} \cdot p_{\text {free }} \cdot p_{\text {stop dom } 2}$

$\hat{P}_{\text {gap } ~}=p_{\text {free } 4}$

$\hat{P}_{2}=\hat{p}_{\text {gtop up }}$ " $\hat{p}_{\text {freg }} \mathbf{s}^{\prime} \hat{p}_{\text {gtop down }}$

$\hat{P}_{\text {gap }:}=p_{\text {free }}$

$\hat{P}_{4}=p_{\text {step up } 4} \cdot p_{\text {free }} \mathrm{y}^{*} p_{\text {step down } 4}$

Therefore, the calculation of the matrix propagation over the quadruple potential barrier is:

$\hat{p}=\hat{P}_{1} \cdot \hat{P}_{g a p 1} \cdot \hat{P}_{2} \cdot \hat{P}_{g a p z} \cdot \hat{P}_{3} \cdot \hat{P}_{g a p z} \cdot \hat{P}_{4}$

The variation of the breakthrough effect results from organizing the arrangement of the semiconductor material as a barrier. The quadruple barrier has 4: a different arrangement or $4 \times 3 \times 2 \times 1=24$. To simplify the analysis, the semiconductor material AlAs is denoted A, GaAs is denoted $\mathrm{B}, \mathrm{GaSb}$ is denoted $\mathrm{C}$, and $\mathrm{InP}$ is denoted D. The arrangements formed are as Figure 4. 


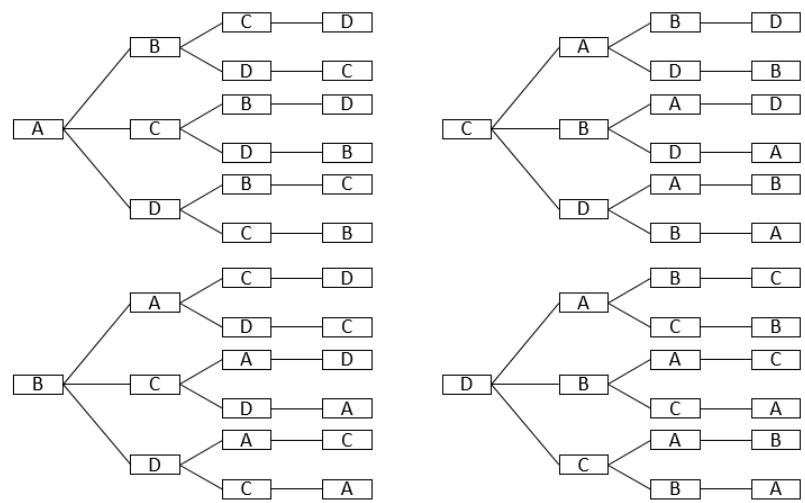

Figure 4. Arrangement variations

\section{Results and Discussion}

The transmission coefficient is analyzed due to variations in the barrier's potential arrangement. The occurrence of the Tunnelling effect is highly dependent on the width and height of the barrier. In this study, the barrier's width was reasonably uniform, ranging from 0.565 to $0.610 \mathrm{~nm}$. Meanwhile, the height of the barrier used was varied, namely three obstacles with a reasonably uniform height $(\mathrm{B}, \mathrm{C}$, and $\mathrm{D})$ and other potential barriers, much higher than the other obstacles (A). These four barriers are the III-V semiconductor alloy. The barrier selection is based on its application, which is widely applied as electronic and optoelectronic devices.

The Tunnelling effect happens when a particle breaks through a potential barrier that classical physics cannot occur. Electrons are represented by the wave function $\Psi$ in each potential region. The wave functions that represent electrons have different shapes in the regions $V=0$ and $V=V_{j}$. In the $V=0$ area, the wave function in the complex exponential form with value $k_{1} x$ is imaginary (0). However, when the electron is in the potential region $V=V_{j}$, the wave function is exponential, and the value $k_{j} x$ is real $j=2,3,4$. This happens because, in the region $V=0$, electrons have material properties. According to de Broglie's postulate, when the electrons break through the barrier, the particle properties will change to wave properties.

Electrons moving from the left direction are free particles that are not affected by potential, so they have momentum as considerable as $\sqrt{2 m E}$ the electrons move towards the first barrier. When an electron encounters a potential region $V_{j}$, it will experience a change in momentum is being the order of the barrier potential $0=1,2,3)$. Electrons that successfully break through the potential barrier will accelerate until their momentum returns to their value $\sqrt{2 m E}$. After passing the last barrier, the transmittance or the number of electrons that have successfully penetrated the four barriers indicated by the transmission coefficient's value can be analyzed.

The use of the matrix propagation method analytically results in the formulation of the transmission coefficient on the tunnelling effect of the quadruple potential barrier, namely

$$
T_{4}=\frac{T_{1}\left|t_{2}\right|^{2}\left|t_{3}\right|^{2}\left|t_{4}\right|^{2}}{\left|1-[\alpha+i \beta] e^{i h_{1}\left(L_{1}+L_{2}+L_{3}{ }^{3}\right.}-\frac{r_{1} r_{4} t_{2} t_{3}}{\tau_{2}{ }^{2} \tau_{3}{ }^{2}} e^{i n h_{1}\left(L_{1}+L_{2}+L_{3} 3\right.}\right|^{2}}
$$

with

$$
\begin{aligned}
& T_{1}=\left(1+\left(\frac{k_{1}^{2}+k_{2}^{2}}{2 k_{1} k_{2}}\right)^{2} \sinh ^{2}\left(k_{2} a\right)\right)^{-1} \\
& \left|t_{2}\right|^{2}=\left(1+\left(\frac{k_{1}^{2}+k_{3}^{2}}{2 k_{1} k_{3}}\right)^{2} \sinh ^{2}\left(k_{3} b\right)\right)^{-1} \\
& \left|t_{2}\right|^{2}=\left(1+\left(\frac{k_{1}^{2}+k_{4}^{2}}{2 k_{1} k_{4}}\right)^{2} \sinh ^{2}\left(k_{4} C\right)\right)^{-1} \\
& \left|t_{4}\right|^{2}=\left(1+\left(\frac{k_{1}^{2}+k_{5}^{2}}{2 k_{1} k_{5}}\right)^{2} \sinh ^{2}\left(k_{5} d\right)\right)^{-1} \\
& t_{1}=\frac{2 k_{1} k_{2}}{2 k_{1} k_{2} \cosh \left(k_{2} a\right)-i\left(k_{1}^{2}-k_{2}^{2}\right) \sinh \left(k_{2} a\right)} \\
& t_{2}=\frac{2 k_{1} k_{3}^{2}}{2 k_{1} k_{3} \cosh \left(k_{3} b\right)-i\left(k_{1}^{2}-k_{3}^{2}\right) \sinh \left(k_{3} b\right)} \\
& t_{3}=\frac{2 k_{1} k_{4}}{2 k_{1} k_{4} \cosh \left(k_{4} c\right)-i\left(k_{1}^{2}-k_{4}^{2}\right) \sinh \left(k_{4} c\right)} \\
& t_{4}=\frac{2 k_{1} k_{5}}{2 k_{1} k_{5} \cosh \left(k_{5} d\right)-i\left(k_{1}^{2}-k_{5}^{2}\right) \sinh \left(k_{5} d\right)} \\
& n_{1}=\frac{\left(k_{1}^{2}+k_{2}^{2}\right) \sinh \left(k_{2} a\right)}{\left(k_{1}^{2}-k_{2}^{2}\right) \sinh \left(k_{2} a\right)+2 \bar{k} k_{1} k_{2} \cosh \left(k_{2} a\right)} \\
& \left(k_{1}^{2}+k_{2}^{2}\right) \sinh \left(k_{2} b\right) \\
& n_{2}=\frac{\left(k_{1}^{2}-k_{3}^{2}\right) \sinh \left(k_{3} b\right)+2 i k_{1} k_{3} \cosh \left(k_{3} b\right)}{\left(k_{1}\right)} \\
& n_{3}=\frac{\left(k_{1}^{2}+k_{4}^{2}\right) \sinh \left(k_{4} c\right)}{\left(k_{1}^{2}-k_{4}^{2}\right) \sinh \left(k_{4} a\right)+2 i k_{1} k_{4} \cosh \left(k_{4} c\right)} \\
& \eta_{4}=\frac{\left(k_{1}^{2}+k_{5}^{2}\right) \sinh \left(k_{5} d\right)}{\left(k_{1}^{2}-k_{5}^{2}\right) \sinh \left(k_{5} d\right)+2 i k_{1} k_{5} \cosh \left(k_{5} d\right)}
\end{aligned}
$$

The use of the matrix propagation method in determining electrons' transmittance is beneficial for many barrier structures because the calculation is sufficient with matrix multiplication and a little mathematical manipulation.

The transmission coefficient is the probability of the particle breaking through a potential barrier $0 \leq T \leq 1$. The tunnelling effect variation is intended to arrange the semiconductor material to form a quadruple potential barrier with different barriers. The barrier is the potential energy of the area that blocks particles' motion when trying to breakthrough. Table 1 showed the transmission coefficient value using Matlab2018a. 
Table 1. The transmission coefficient of the quadruple potential barrier

\begin{tabular}{|c|c|c|c|c|c|c|}
\hline \multirow{2}{*}{ E (eV) } & \multicolumn{6}{|c|}{$\mathbf{T}$} \\
\hline & ABCD & ABDC & ACBD & ACDB & ADBC & ADCB \\
\hline 0 & 0.0000 & 0.0000 & 0.0000 & 0.0000 & 0.0000 & 0.0000 \\
\hline 0.1 & 0.0001 & 0.0001 & 0.0000 & 0.0000 & 0.0000 & 0.0000 \\
\hline 0.25 & 0.0000 & 0.0007 & 0.0008 & 0.0008 & 0.0007 & 0.0008 \\
\hline 0.5 & 0.2551 & 0.0607 & 0.6035 & 0.7695 & 0.0618 & 0.3000 \\
\hline 0.506 & 0.2502 & 0.0681 & 0.7100 & 0.8054 & 0.0697 & 0.2837 \\
\hline 0.518 & 0.2284 & 0.0851 & 0.8009 & 0.7082 & 0.0885 & 0.2434 \\
\hline 0.733 & 0.1205 & 0.3232 & 0.1669 & 0.1539 & 0.3613 & 0.1200 \\
\hline 0.75 & 0.1301 & 0.3317 & 0.1760 & 0.1644 & 0.3650 & 0.1303 \\
\hline 0.76 & 0.1368 & 0.3376 & 0.1825 & 0.1718 & 0.3683 & 0.1375 \\
\hline \multirow[t]{2}{*}{1} & 0.7711 & 0.5466 & 0.5058 & 0.5547 & 0.5064 & 0.8087 \\
\hline & BACD & BADC & BCAD & BCDA & BDAC & BDCA \\
\hline 0 & 0.0000 & 0.0000 & 0.0000 & 0.0000 & 0.0000 & 0.0000 \\
\hline 0.1 & 0.0001 & 0.0001 & 0.0001 & 0.0000 & 0.0001 & 0.0000 \\
\hline 0.25 & 0.0007 & 0.0007 & 0.0007 & 0.0008 & 0.0006 & 0.0008 \\
\hline 0.5 & 0.0980 & 0.0274 & 0.0981 & 0.3000 & 0.0290 & 0.7695 \\
\hline 0.506 & 0.1120 & 0.0299 & 0.1132 & 0.2837 & 0.0321 & 0.8054 \\
\hline 0.518 & 0.1442 & 0.0357 & 0.1496 & 0.2434 & 0.0396 & 0.7082 \\
\hline 0.733 & 0.1831 & 0.2981 & 0.2021 & 0.1200 & 0.6991 & 0.1539 \\
\hline 0.75 & 0.1849 & 0.3208 & 0.2023 & 0.1303 & 0.6904 & 0.1644 \\
\hline 0.76 & 0.1870 & 0.3341 & 0.2035 & 0.1375 & 0.6794 & 0.1718 \\
\hline \multirow[t]{2}{*}{1} & 0.4404 & 0.7329 & 0.4267 & 0.8087 & 0.5026 & 0.5547 \\
\hline & CABD & CADB & CBAD & CBDA & CDAB & CDBA \\
\hline 0 & 0.0000 & 0.0000 & 0.0000 & 0.0000 & 0.0000 & 0.0000 \\
\hline 0.1 & 0.0001 & 0.0001 & 0.0001 & 0.0001 & 0.0001 & 0.0001 \\
\hline 0.25 & 0.0006 & 0.0006 & 0.0007 & 0.0007 & 0.0007 & 0.0007 \\
\hline 0.5 & 0.0264 & 0.0290 & 0.0251 & 0.0618 & 0.0274 & 0.0607 \\
\hline 0.506 & 0.0291 & 0.0321 & 0.0274 & 0.0697 & 0.0299 & 0.0681 \\
\hline 0.518 & 0.0355 & 0.0396 & 0.0326 & 0.0885 & 0.0357 & 0.0851 \\
\hline 0.733 & 0.6592 & 0.6991 & 0.3244 & 0.3613 & 0.2981 & 0.3232 \\
\hline 0.75 & 0.6777 & 0.6904 & 0.3532 & 0.3650 & 0.3208 & 0.3317 \\
\hline 0.76 & 0.6805 & 0.6794 & 0.3697 & 0.3683 & 0.3341 & 0.3376 \\
\hline \multirow[t]{2}{*}{1} & 0.5467 & 0.5026 & 0.7575 & 0.5064 & 0.7329 & 0.5466 \\
\hline & DABC & DACB & DBAC & DBCA & DCAB & DCBA \\
\hline 0 & 0.0000 & 0.0000 & 0.0000 & 0.0000 & 0.0000 & 0.0000 \\
\hline 0.1 & 0.0001 & 0.0001 & 0.0001 & 0.0000 & 0.0001 & 0.0000 \\
\hline 0.25 & 0.0007 & 0.0007 & 0.0006 & 0.0008 & 0.0007 & 0.0003 \\
\hline 0.5 & 0.0251 & 0.0981 & 0.0264 & 0.6035 & 0.0980 & 0.2551 \\
\hline 0.506 & 0.0274 & 0.1132 & 0.0291 & 0.7100 & 0.1120 & 0.2502 \\
\hline 0.518 & 0.0326 & 0.1496 & 0.0355 & 0.8009 & 0.1442 & 0.2284 \\
\hline 0.733 & 0.3244 & 0.2021 & 0.6592 & 0.1669 & 0.1831 & 0.1205 \\
\hline 0.75 & 0.3532 & 0.2023 & 0.6777 & 0.1760 & 0.1849 & 0.1301 \\
\hline 0.76 & 0.3697 & 0.2035 & 0.6805 & 0.1825 & 0.1870 & 0.1368 \\
\hline 1 & 0.7575 & 0.4267 & 0.5467 & 0.5058 & 0.4404 & 0.7711 \\
\hline
\end{tabular}

The results show that the inverted arrangement produces the same transmission coefficient value for each energy. There are 12 kinds of transmission coefficient values generated from 24 arrangements. Even though the 24 arrays consist of the same four semiconductor materials, it turns out that different arrangements produce different transmission coefficient values, except for the reverse arrangement, which results in the same transmission coefficient value for each energy. It can be observed in Table 1 that an arrangement that has identical transmission coefficients are $\mathrm{ABCD}$ with DCBA, CDBA with ABDC, etc.

Based on Table 1, it can be analyzed that the increase of the electron energy resulting in increased electron transmittance. The simulation results show that the transmission coefficient increases with increasing electron energy until it reaches the maximum value but decreases. This decrease occurs because the transmission coefficient's essential function is hyperbolic trigonometry, which causes the transmission coefficient to be periodic. At some point, the value will reach a maximum, then drop to a minimum, and rise again.

According to the Figure 5, the composition of semiconductor materials with the most considerable transmission coefficient value is $\mathrm{ADCB}$ and $\mathrm{BCDA}$, valued at 0.8087 . This means that $80.87 \%$ of the electrons can pass through the potential barrier. The arrangement of ADCB and BCDA has a reasonably large coefficient value than other arrangements because the three potential barriers whose potential heights are quite symmetrical are close together. This arrangement of the barrier causes a resonance so that the probability of the electron passing is high. However, this probability cannot reach the maximum because the electrons have to return through the potentially high barrier.

From the Figure 6, the smallest transmission coefficient value generated by the BCAD and $\mathrm{DACB}$ arrangements is 0.4267 . This means that only $42.67 \%$ of the electrons can pass through the potential barrier. In this arrangement, the symmetrical potential barrier is separated by one barrier, which has enormous potential. Furthermore, the adjacent barrier is not symmetrical enough so that the resonance phenomenon that occurs cannot be maximized. Therefore, it can be analyzed that one potential barrier affects another potential barrier. The variation of the arrangement affects the value of the transmission coefficient so that it can be used as a guideline for selecting the arrangement that produces the most optimum value of the transmission coefficient from various possible arrangements.
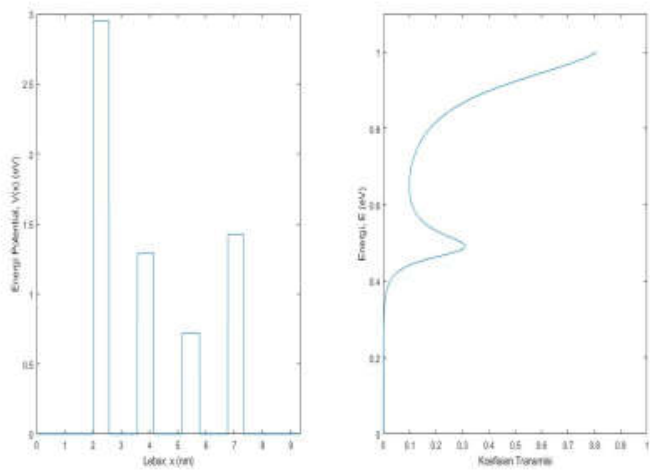

Figure 5. ADCB arrangement that produces the maximum transmission coefficient value
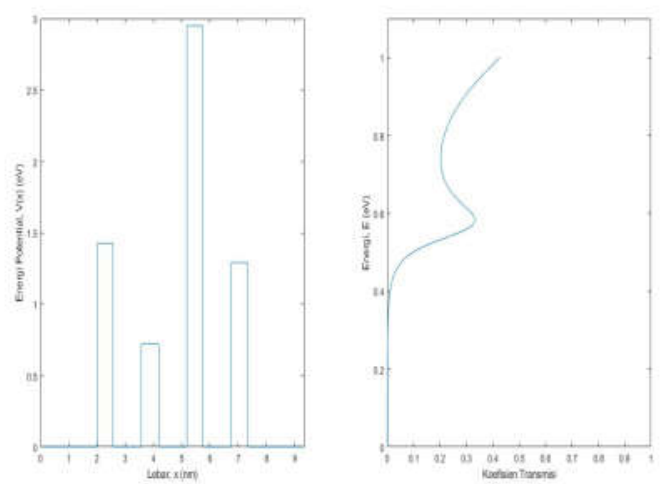

Figure 6. BCAD arrangement that produces the minimum transmission coefficient value 


\section{Conclusion}

The transmission coefficient of the quadruple potential barrier using the matrix propagation method can be formulated as follows.

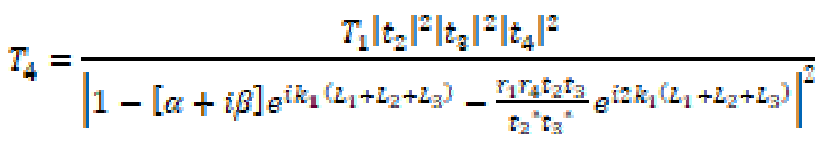

The variation of the Tunnelling effect on the quadruple barrier potential is as many as 24 arrays. The research shows that 24 potential arrangements of the quadruple barrier produce 12 variations in the value of the transmission coefficient, with the reverse arrangement resulting in the same transmission coefficient value for each energy. The semiconductor material composition with the enormous transmission coefficient value is $\mathrm{ADCB}$ and BCDA, which have a value of 0.8087 . Moreover, the smallest transmission coefficient value generated by the BCAD and DACB arrangement is a value of 0.4267 . This variation of the breakthrough effect occurs because one potential barrier affects another potential barrier.

\section{Acknowledgment}

We gratefully acknowledge the Research Group of Department Physics Education from FKIP-University of Jember.

\section{References}

[1] R. A. Sani, M. Kadri, Quantum Physics. Jakarta: Bumi Aksara, 2016.

[2] R. M. Eisenberg and R. Resnick, Quantum Physics of Atoms, Molecules, Solids, Nuclei and Particles. New York: John Wiley \& Sons, 1985.

[3] I. Abdulhalim, "Analytic Propagation Matrix Method for Anisotropic Magneto-Optic Layered Media," J. Opt. A Pure Appl. Opt., vol. 2, no. 6, pp. 557-564, 2000.

[4] A. F. J. Levi and W. R. Frensley, Applied Quantum Mechanics. Cambridge: Cambridge University Press, 2003.

[5] S. D. G. Martinz and R. V. Ramos, "Double Quantum Well Triple Barrier Structures: Analytical and Numerical Results," Can. J. Phys., vol. 94, no. 11, pp. 1180-1188, 2016.
[6] B. Supriadi, Z. R. Ridlo, Yushardi, C. I. W. Nugroho, J. Arsanti, and S. Septiana, "Tunnelling effect on triple potential barriers GaN, SiC and GaAs," J. Phys. Conf. Ser., vol. 1211, no. 1, 2019.

[7] S. H. B. Prastowo, B. Supriadi, Z. R. Ridlo, M. K. Huda, W. Bariroh, and U. Sholihah, "Theoretical Analysis Quantum Tunnelling Three Potential Barriers to the Schrodinger Equation in Grapheme," J. Phys. Conf. Ser., vol. 1211, no. 1, 2019.

[8] R. E. Siregar, "Quantum Physics," in Quantum Physics, Jatinangor: Universitas Padjajaran Press, 2018.

[9] A. Purwanto, Quantum Physics 2nd Revised Edition. Yogyakarta: Penerbit Gava Media, 2016.

[10] K. S. Krane, Modern Physics Translation by Wospakrik H. J and Niksolihin S. Jakarta: UIP, 1992.

[11] Sujarwata and P. Marwoto, Thin Film Characterization: Theory, Application and Analysis of Thin Film Deposition Results. Yogyakarta: Deepublish, 2014.

[12] G. Chen, Nanoscale Energy Transport and Conversion: A Parallel Treatment of Electrons, Molecules, Phonons, and Photons. Oxford: Oxford University Press, 2005.

[13] Jorena, "Determining the Energy Gap of Silicon Semiconductors through Measurement of Material Resistance at Various Temperatures," J. Penelit. Sains, vol. 12, no. 1, pp. 1-3, 2009.

[14] S. H. B. Prastowo, B. Supriadi, Z. R. Ridlo, and T. Prihandono, "Tunnelling Effect on Double Potential Barriers GaAs and PbS," J. Phys. Conf. Ser., vol. 1008, no. 1, 2018.

[15] A. G. Milnes and A. Y. Polyakov, "Gallium Antimonide Device Related Properties," Solid State Electron., vol. 36, no. 6, pp. 803-818, 1993.

[16] A. Sasaki, M. Nishiuma, and Y. Takeda, "Energy Band Structure and Lattice Constant Chart of III-V Mixed Semiconductors, and AlGaSb/AlGaAsSb Semiconductor Lasers on GaSb Substrates," Jpn. J. Appl. Phys., vol. 19, no. 9, pp. 1695-1702, 1980.

[17] P. S. Dutta, H. L. Bhat, and V. Kumar, "The physics and Technology of Gallium Antimonide: An Emerging Optoelectronic Material," J. Appl. Phys., vol. 81, no. 9, pp. 5821-5870, 1997.

[18] J. J. Coleman, P. D. Dapkus, N. Holonyak, and W. D. Laidig, "Device-quality Epitaxial AlAs by Metalorganicchemical Vapor Deposition," Appl. Phys. Lett., vol. 38, no. 11, pp. 894-896, 1981.

[19] S. Adachi, GaAs and Related Materials: Bulk Semiconducting and Superlattice Properties. Singapore: World Scientific Publishing Co. Pte. Ltd, 1994.

[20] A. Mikrajuddin, Nanomaterial Characterization. Bandung: CV Rezeki Putera, 2010. 